\title{
R-Generalized Fuzzy Closed Sets with Respect to an Ideal in Fuzzy Topological Spaces
}

\section{Saber MY*}

Department of Mathematics, Faculty of Science, Al-Azhar University, Assiut, Egypt

Department of Mathematics, Faculty of Science and Humanities, Majmaah University, Riyadh, Saudi-Arabia

\begin{abstract}
The work in this paper is a generalization The concept of r-generalized fuzzy closed sets in fuzzy topological spaces was introduced by Kim. In this paper, we introduce and study the concept of r-generalized fuzzy closed sets with respect to an ideal in an ideal fuzzy topological space in Sostak sense.
\end{abstract}

Keywords: R-generalized fuzzy closed sets; Rv-generalized fuzzy closed sets with respect to an ideal in an ideal fuzzy topological space in Sostak sense

\section{Introduction}

Sostak introduce the fundamental concept of fuzzy topological structure as an extension of both crisp topology and Chang's fuzzy topology [1], in the sense that not only the object was fuzzified, but also the axiomatic. Chattopdhyay et al. [2,3] have redefined the similar concept. In El-Naschie [4-14] and Kim and Ko [15] gave a similar definition namely "Smooth fuzzy topology". We must point out that [16-19]; the concept of fuzzy topological spaces has been a significant concept in string theory and E-infinity theory pertaining to quantum particular physics ever since El-Naschie ([4-14]). After that several authors $[20,21]$ have introduced the smooth definition and studied smooth fuzzy idea topological spaces being unaware of Ŝstak works.

Throughout this paper, let $\mathrm{X}$ be a nonempty set $\mathrm{I}=[0 ; 1]$ and $\mathrm{I}_{0}=(0 ; 1]$ : For $\alpha \in \mathrm{I} ; \bar{\alpha}(x)=\alpha$ for all $\mathrm{x} \in \mathrm{X}$ : The family of all fuzzy sets on $\mathrm{X}$ denoted by $\mathrm{I}^{\mathrm{X}}$ : For two fuzzy sets we write $\lambda_{\text {qu }}$ to mean that is quasicoincident ( $\mathrm{q}$-coincident, for short) with $\mu$, i.e., there exists at least one point $\mathrm{x} \in \mathrm{X}$ such that $\lambda(\mathrm{x})+\mu(\mathrm{x})>1$ : Negation of such a statement is denoted as $\lambda_{\bar{q} \mu}$ :

\section{Definition 1.1}

A mapping $\tau$ : $\mathrm{I}^{\mathrm{X}} \rightarrow \mathrm{I}$ is called a fuzzy topology on $\mathrm{X}$ if it satisfies the following conditions [17]:

$$
\begin{aligned}
& \tau(\overline{0})=\tau(\overline{1})=\overline{1} \\
& \tau\left(\vee_{i \in \Gamma} \mu_{i}\right) \geq \wedge_{i \in \Gamma} \tau\left(\mu_{i}\right), \text { for any }\left\{\mu_{i}\right\}_{i \in \Gamma} \in I^{X} \\
& \tau\left(\mu_{1} \wedge \mu_{2}\right) \geq \tau\left(\mu_{1}\right) \wedge \tau\left(\mu_{2}\right) \text { for any } \mu_{1}, \mu_{2} \in I^{X}
\end{aligned}
$$

The pair $(X ; \tau)$ is called a fuzzy topological space (for short, $\mathrm{fts}$ ).

\section{Definition 1.2}

Let $(\mathrm{X}, \tau)$ be a fts, $\lambda, \mu \in \mathrm{I}^{\mathrm{X}}$ and $\mathrm{r} \in \mathrm{I}_{0}$.

A fuzzy set $\lambda$ is called r-generalized fuzzy closed (for short, r-gfc) if $\mathrm{C}_{\gamma}(\lambda ; \gamma)$ whenever $\lambda \leq \mu$ and $\tau(\mu) \geq \gamma$

A fuzzy set $\lambda$ is called $r$-generalized fuzzy closed (for short, $r$-gfc) if $I_{\tau}(\lambda ; \gamma) \geq \mu$ whenever $\lambda \geq \mu$ and $\tau(\overline{1}-\mu) \geq \gamma$

\section{Definition 1.3}

A mapping $\mathrm{I}: \mathrm{I}^{\mathrm{X}} \rightarrow \mathrm{I}$ is called fuzzy ideal on $\mathrm{X}$ if :
$\left(\mathrm{I}_{1}\right) \mathrm{I}(0)=1 ; \mathrm{I}(1)=0$ :

(I $\left.{ }_{2}\right)$ If $\lambda \leq \mu$; then $\mathrm{I}(\lambda) \geq \mathrm{I}(\lambda)$; for each $\lambda \in \mathrm{I}^{\mathrm{X}}$ :

$\left(\mathrm{I}_{3}\right)$ For each $\lambda ; \mu \in \mathrm{I}^{\mathrm{X}} ; \mathbf{I}(\lambda \mathrm{v} \mu) \geq \mathbf{I}(\lambda) \wedge \mathbf{I}(\mu)[$ finite additivity].

\section{Lemma 1.1.}

Let $(\mathrm{X}, \tau, \mathrm{I})$ be a fits. The simplest fuzzy ideal on $\mathrm{X}$ are $\mathrm{I}^{0}, \mathrm{I}^{1}: \mathrm{I}^{\mathrm{X}} \rightarrow \mathrm{I}$ where

$$
I^{0}(\lambda)=\left\{\begin{array}{l}
1, \text { if } \lambda=\underline{0} \\
0, \text { otherwise, }
\end{array} \quad I^{1}(\lambda)=\left\{\begin{array}{l}
0, \text { if } \lambda=\underline{1} \\
1, \text { otherwise }
\end{array} .\right.\right.
$$

If we take $\mathrm{I}=\mathrm{I}^{0}$, for each $\mathrm{A} \in \mathrm{I}^{\mathrm{X}}$ we have $\mathrm{A}_{\mathrm{r}}^{*}=\mathrm{C}_{\tau}(\mathrm{A}, \mathrm{r})$.

If we take $\mathrm{I}=\mathrm{I}^{1}$, for each $\mathrm{A} \in \Theta^{\prime}$ we have $A^{*}{ }_{r}=0$, where, $\underline{1} \notin \Theta^{\prime}$ be a subset of $\mathrm{I}^{\mathrm{X}}[4-14]$.

\section{Definition 1.4}

Let $(X, \tau, I)$ be a fuzzy ideal topological space[16]. Let $\mu, \lambda \in \mathrm{I}^{\mathrm{X}}$, the r-fuzzy open local function $\mu_{r}^{*}$ of $\mu$ is the union of all fuzzy points $x_{t}$ such that if $\rho \in Q\left(x_{t}, \gamma\right)$ and $I(\lambda) \geq r$ then there is at least one $y \in X$ for which $\rho(y)+\mu(y)-1>\lambda(y)$.

\section{Theorem 1.1}

Let $(\mathrm{X}, \tau)$ be a fts. Then for each $\mathrm{r} \in \mathrm{I}_{0}, \lambda \in \mathrm{I}^{\mathrm{X}}$ we define an operator $\mathrm{C}_{\tau}: \mathrm{I}^{\mathrm{X}} \times \mathrm{I}_{0} \rightarrow \mathrm{I}^{\mathrm{X}}$ as follows:

$$
C_{\tau}(\lambda, \gamma)=\wedge\left\{\mu \in I^{x}: \lambda \leq \mu, \tau(\overline{1}-\mu) \geq \gamma\right\}
$$

For $\lambda, \mu \in \mathrm{I}^{\mathrm{X}}$ and $\mathrm{r}, s \in \mathrm{I}_{0}$, the operator $\mathrm{C}_{\tau}$ satisfies the following conditions:

$$
\begin{aligned}
& \mathrm{C} \tau(\overline{0}, \gamma)=\overline{0} \\
& \lambda \leq \mathrm{C} \tau(\lambda, \gamma)
\end{aligned}
$$

*Corresponding author: Saber MY, Department of Mathematics, Faculty of Science and Humanities, Majmaah University, Riyadh 11982, Saudi Arabia, Tel: +966 16404 4444; E-mail: m.ah75@yahoo.com

Received March 28, 2018; Accepted April 04, 2018; Published April 15, 2018

Citation: Saber MY (2018) R-Generalized Fuzzy Closed Sets with Respect to an Ideal in Fuzzy Topological Spaces. J Appl Computat Math 7: 392. doi: 10.4172/2168-9679.1000392

Copyright: @ 2018 Saber MY. This is an open-access article distributed under the terms of the Creative Commons Attribution License, which permits unrestricted use, distribution, and reproduction in any medium, provided the original author and source are credited. 


$$
\begin{aligned}
& \mathrm{C} \tau(\lambda, \gamma) \vee \mathrm{C} \tau(\mu, \gamma)=\mathrm{C} \tau(\lambda \vee \mu, \gamma) \\
& \mathrm{C} \tau(\lambda, \gamma) \vee \mathrm{C} \tau(\lambda, \mathrm{s}) \text { if } \gamma \leq s \\
& \mathrm{C} \tau(\mathrm{C} \tau(\lambda, \gamma), \gamma)=\mathrm{C} \tau(\lambda, \gamma)
\end{aligned}
$$

\section{Theorem 1.2}

Let $(\mathrm{X}, \tau)$ be a fts. Then for each $\mathrm{r} \in \mathrm{I}_{0}, \lambda \in \mathrm{I}^{\mathrm{X}}$ we define an operator $\mathrm{I} \tau: \mathrm{I}^{\mathrm{X}} \times \mathrm{I}_{0} \rightarrow \mathrm{I}^{\mathrm{X}}$ as follows [18]:

$$
I \tau\left((\lambda, \gamma)=\vee\left\{\mu \in \mathrm{I}^{x}: \lambda \geq \mu, \tau(\mu) \geq \gamma\right\}\right.
$$

For $\lambda, \mu \in \mathrm{I}^{\mathrm{X}}$ and $\mathrm{r}, \mathrm{s} \in \mathrm{I}_{0}$, the operator $\mathrm{I}_{\tau}$ satisfies the following conditions:

$$
\begin{aligned}
& I_{\tau}\left((\overline{1}-\lambda, \gamma)=\overline{1}-C_{\tau}(\lambda, \gamma\} \text { and } C_{\tau}(\overline{1}-\lambda, \gamma)=\overline{1}-I_{\tau}(\lambda, \gamma)\right. \\
& I_{\tau}(\overline{1}, \gamma)=\overline{1} . \\
& \lambda \geq I_{\tau}(\lambda, \gamma) \\
& I_{\tau}(\lambda, \gamma) \wedge I_{\tau}\{\mu, \gamma\}=I_{\tau}(\lambda \wedge \mu, \gamma) \\
& I_{\tau}(\lambda, \gamma) \wedge I_{\tau}\{\lambda, s\} \text { if } \gamma \geq \mathrm{s} . \\
& I_{\tau}\left(I_{\tau}(\lambda, \gamma), \gamma\right)=I_{\tau}\{\lambda, \gamma\}
\end{aligned}
$$

\section{r-generalized fuzzy closed sets with respect to an ideal}

\section{Definition 2.1}

Let $(X, \tau, I)$ be fuzzy ideal topological space, $\mu \in I^{X}$ and $r \in I_{0}$. A fuzzy set $\mu$ is called $r$-generalized fuzzy closed with respect to an ideal I (briefly, r-gfIc) if $\mathbf{I}\left(\mathrm{C}_{\tau}(\mu, \gamma) \mid \lambda\right) \geq \gamma$, whenever $\mu \leq \lambda$ and $\tau(\lambda) \geq \mathrm{r}$.

\section{Lemma 2.2}

Every r-gfc set is r-gfIc.

\section{Proof}

Let $\mu \leq \lambda$ and $\tau(\lambda) \geq$ r. Since $\mu$ is r-gfc set, then $C \tau(\mu, \gamma) \leq \lambda$, this implies that $C \tau(\mu, \gamma) \bar{q} \underline{1}-\lambda$, implies $C_{\tau}(\mu, r)(x)+(\underline{1}-\lambda)(x) \leq 1$, then $\mathrm{C}_{\tau}(\mu, \gamma)(\mathrm{x})-\lambda(\mathrm{x}) \leq 0$. Thus, $\mathrm{I}\left(\mathrm{C}_{\tau}(\mu, \mathrm{r}) \backslash \lambda\right) \geq \gamma[16-19]$.

\section{Example}

The converse Lemma 2.2 is not true. Let $X=\{a, b\}$ be a set.

$\mu_{1}(a)=0.4, \mu_{1}(b)=0.5 ; \mu_{2}(a)=0.4 \mu_{2}(b)=0.6 ; \mu_{1}(a)=0.3, \mu_{1}(b)=0.5$.

We define fuzzy topology and fuzzy ideal $\tau, \mathrm{I}: \mathrm{I}^{\mathrm{X}} \rightarrow \mathrm{I}$ as follows

$$
\tau(\lambda)=\left\{\begin{array}{l}
1, \text { if } \lambda=\underline{1}, \underline{0} \\
\frac{1}{2}, \text { if } \lambda=\mu_{1}, \\
\frac{1}{2}, \text { if } \lambda=\mu_{2} \\
0, \text { otherwise }
\end{array} \mathbb{J}(\lambda)=\left\{\begin{array}{l}
1, \text { if } \lambda=\underline{0} \\
\frac{1}{2}, \text { if } \lambda=\underline{0} .5 \\
\frac{1}{2}, \text { if } \underline{0}<\lambda<\underline{0} .5 \\
0, \text { otherwise }
\end{array}\right.\right.
$$

Then $\mu$ is r-gflc set because,

$$
\mu \leq \mu_{1}, \tau\left(\mu_{1}\right) \geq \frac{1}{2} C_{\tau}\left(\mu, \frac{1}{2}\right)=\underline{1}-\mu_{1} \backslash \mu_{1}=a_{0.3} .
$$

\section{Theorem 2.1}

Let $(\mathrm{X}, \tau, \mathrm{I})$ be an fuzzy ideal topological space, $\mu, \lambda \in \mathrm{I}^{\mathrm{X}}$ and $\mathrm{r} \in \mathrm{I}_{0}$. If $\mu$ and $\lambda$ are $r$-gfIc sets, then $\mu \mathrm{V} \lambda$ is r-gfIc.

\section{Proof}

Suppose $\mu$ and $\lambda$ are r-gfIc sets. If $\mu \vee \lambda \leq \rho$ and $\tau(\rho) \geq \gamma$, then $\mu \leq$ $\rho$ and $\lambda \leq \rho$. By assumption, $\mathrm{I}\left(\mathrm{C}_{\tau}(\mu, \gamma) \backslash \rho\right) \geq \gamma$ and $\mathrm{I}\left(\mathrm{C}_{\tau}(\lambda, \gamma) \backslash \rho\right) \geq \gamma$ and hence

$$
\mathbf{I}\left(\mathrm{C}_{\tau}(\mu \vee \lambda, \gamma) \backslash \rho=\mathrm{C} \tau(\mu, \gamma) \backslash \rho \vee C \tau(\lambda, \gamma) \backslash \rho\right) \geq \gamma .
$$

Therefore, $\mu \mathrm{V} \lambda$ is $\mathrm{r}$-gfIc.

\section{Remark}

The intersection of two r-gflc sets need not be an r-gfIc set as shown by the following example.

\section{Example}

The converse Lemma 2.2 is not true. Let $\mathrm{X}=\{\mathrm{a}, \mathrm{b}\}$ be a set.

$\mu_{1}(a)=0.4, \mu_{1}(b)=0.5 ; \mu_{2}(a)=0.4 \mu_{2}(b)=0.6 ; \mu_{1}(a)=0.3, \mu_{1}(b)=0.5$.

We define fuzzy topology and fuzzy ideal $\tau, \mathbf{I}: \mathrm{I}^{\mathrm{X}} \rightarrow \mathrm{I}$ as follows:

$$
\tau(\lambda)=\left\{\begin{array}{l}
1, \text { if } \lambda=\underline{1}, \underline{0} \\
\frac{1}{2}, \text { if } \lambda=\mu_{1}, \\
\frac{1}{2}, \text { if } \lambda=\mu_{2} \\
0, \text { otherwise }
\end{array} \mathbb{J}(\lambda)=\left\{\begin{array}{l}
1, \text { if } \lambda=\underline{0} \\
\frac{1}{2}, \text { if } \lambda=\underline{0} .5 \\
\frac{1}{2}, \text { if } \underline{0}<\lambda<\underline{0} .5 \\
0, \text { otherwise }
\end{array}\right.\right.
$$

Then $\mu$ is r-gflc set because,

$$
\mu \leq \mu_{1}, \tau\left(\mu_{1}\right) \geq \frac{1}{2} C_{\tau}\left(\mu, \frac{1}{2}\right)=\underline{1}-\mu_{1} \backslash \mu_{1}=a_{0.3}
$$

Therefore,

$$
\mathbf{I}\left(C \tau\left(\mu, \frac{1}{2}\right) \backslash \mu_{1}\right) \geq \frac{1}{2} \text {. }
$$

But $\mu$ is not $r$-gfc set because

$$
\mu \leq \mu_{1}, \tau\left(\mu_{1}\right) \geq \frac{1}{2} C_{\tau}\left(\mu, \frac{1}{2}\right)=\underline{1}-\mu_{1} \nless \mu_{1}
$$

\section{Theorem 2.2}

Let $(\mathrm{X}, \tau, \mathrm{I})$ be an fuzzy ideal topological space, $\mu, \lambda \in \mathrm{I}^{\mathrm{X}}$ and $\gamma \in \mathrm{I}_{0}$. If $\mu$ is $r$-gflc set and $\mu \leq \lambda \leq \mathrm{C}_{\tau}(\mu, \gamma)$, then $\lambda$ are $\mathrm{r}$-gfIc.

\section{Proof}

Let $\mu$ is r-gflc set and $\mu \leq \lambda \leq \mathrm{C}_{\tau}(\mu, \gamma)$. Suppose $\lambda \leq \rho$ and $\tau(\rho) \geq \gamma$. Then $\mu \leq \rho$. Since $\mu$ is r-gfIc, we have $\mathrm{I}\left(\mathrm{C}_{\tau}(\mu, \gamma) \backslash \rho\right) \geq \gamma$. Now $\lambda \leq \mathrm{C}_{\tau}(\mu, \gamma)$ implies that

$$
\mathrm{C}_{\tau}(\lambda, \gamma) \backslash \rho \leq \mathrm{C}_{\tau}(\mu, \gamma) \backslash \rho,
$$

and hence, $I\left(C_{\tau}(\lambda, \gamma) \backslash \rho\right) \geq r$. Therefore, $\lambda$ is r-gfIc set $[20,21]$.

\section{Definition 2.2}

Let $(\mathrm{X}, \tau, \mathrm{I})$ be fuzzy ideal topological space, $\mu \in \mathrm{I}^{\mathrm{X}}$ and $\gamma \in \mathrm{I}_{0}$. A fuzzy set $\mu$ is called $r$-fuzzy generalized open with respect to an ideal I (briefly, $\mathrm{r}$-gflo) if $\underline{1}-\mu$ is $\mathrm{r}$-gfIc set.

\section{Theorem 2.3}

Let $(X, \tau, I)$ be an fuzzy ideal topological space, $\mu, \lambda, \rho \in \mathrm{I}^{\mathrm{X}}$ and $\gamma \in \mathrm{I}_{0}$. If $\mu$ is r-gflo sets if and only if $\lambda \backslash \rho \leq \operatorname{Int} \tau(\mu, r)$ for some $\mathbf{I}(\rho) \geq \mathrm{r}$, whenever $\lambda \leq \mu$ and $\tau(\underline{1}-\lambda) \geq \gamma$.

\section{Proof}

Suppose that $\mu$ is r-gflo sets. Suppose $\lambda \leq \mu$ and $\tau(\underline{1}-\lambda) \geq \gamma$. We have $\underline{1}-\lambda \geq \underline{1}-\mu$. By assumption, 
Citation: Saber MY (2018) R-Generalized Fuzzy Closed Sets with Respect to an Ideal in Fuzzy Topological Spaces. J Appl Computat Math 7: 392. doi: $10.4172 / 2168-9679.1000392$

Page 3 of 4

$$
C_{\tau}(\underline{1}-\mu, \gamma) \leq \underline{1} \vee \rho
$$

For some $\mathbf{I}(\rho) \geq \gamma$. This implies

$$
\underline{1}-((\underline{1}-\lambda) \vee \rho) \leq \underline{1}-C_{\tau}(\underline{1}-\mu)
$$

and hence, $\lambda \backslash \rho \leq \operatorname{Int}_{\tau}(\mu, \gamma)$.

Conversely, assume that $\lambda \leq \mu$ and $\tau(1-\lambda) \geq \gamma$ imply $\lambda \mid \rho \leq \operatorname{Int}_{\tau}(\mu, \gamma)$ for some $\mathrm{I}(\rho) \geq \gamma$. Consider $\tau(\omega) \geq \gamma$ such that $\underline{1}-\mu \leq \omega$. Then $\underline{1}-\omega \leq \mu$. By assumption,

$$
\underline{1}-\omega \backslash \rho \leq \operatorname{Int}_{\tau}(\mu, \gamma)=\underline{1}-C_{\tau}(\underline{1}-\mu, \gamma)
$$

for some $\mathbf{I}(\rho) \geq \gamma$. This gives that $\underline{1}-(\omega \vee \rho) \leq \underline{1}-C_{\tau}(\underline{1}-\mu, \gamma)$. Therefore, $C_{\tau}(\underline{1}-\mu, \gamma) \leq \omega \vee \rho$ for some $\mathrm{I}(\rho) \geq \gamma$. This show that $\mathbf{I}\left(C_{\tau}(\underline{1}-\mu, \gamma) \backslash \omega\right) \geq \gamma$. Hence, $\underline{1}-\mu$ is r-gfIc set.

Recall that the sets $\mu$ and $\lambda$ are fuzzy separated if $C_{\tau}(\mu, \gamma) \bar{q} \lambda$ and $\mu \bar{q} C_{\tau}(\lambda, \gamma)$.

\section{Theorem 2.4}

Let $(X, \tau, I)$ be an fuzzy ideal topological space, $\mu, \lambda, \in \mathrm{I}^{\mathrm{X}}$ and $\mathrm{r} \in \mathrm{I}_{0}$. If $\mu$ and $\lambda$ are fuzzy separated and $r$-gflo sets, then $\mu \mathrm{V} \lambda$ is $r$-gflo.

\section{Proof}

Suppose $\mu$ and $\lambda$ are fuzzy separated and r-gflo sets and $\rho \leq \mu \mathrm{V}$ $\lambda$, and $\tau(\underline{1}-\rho) \geq \gamma$. Then $\rho \wedge \mathrm{C}_{\tau}(\mu, \gamma) \leq \mu$ and $\rho \wedge \mathrm{C} \tau(\lambda, \gamma) \leq \lambda$. By assumption,

$$
\rho \wedge \mathrm{C}_{\tau}(\mu, \gamma) \backslash v_{1} \leq \operatorname{Int}_{\tau}(\mu, \gamma), \rho \wedge \mathrm{C}_{\tau}(\lambda, \gamma) \backslash \nu_{2} \leq \operatorname{Int}_{\tau}(\lambda, \gamma),
$$

for some $\mathbf{I}\left(\nu_{1}, v_{2}\right) \geq \gamma$. This means $\mathbf{I}\left(\rho \wedge \mathrm{C}_{\tau}(\mu, \gamma) \backslash \operatorname{Int}_{\tau}(\mu, \gamma)\right) \geq \gamma$, and $\mathrm{I}\left(\rho \wedge \mathrm{C}_{\tau}(\lambda, \gamma) \backslash \operatorname{Int}_{\tau}(\lambda, \gamma)\right) \geq \gamma$. Thus, $\mathrm{I}\left(\rho \wedge \mathrm{C}_{\tau}(\mu, \gamma) \backslash \operatorname{Int}_{\tau}(\mu, \gamma)\right) \vee\left(\rho \wedge \mathrm{C}_{\tau}(\lambda, \gamma) \backslash\right.$ $\left.\operatorname{Int}_{\tau}(\lambda, \gamma)\right) \geq \gamma$.

\section{Therefore,}

$\mathrm{I}\left(\rho \wedge\left(\mathrm{C}_{\tau}(\mu, \gamma) \mathrm{VC}_{\tau}(\lambda, \gamma)\right) \backslash\left(\operatorname{Int}_{\tau}(\mu, \gamma) \operatorname{VInt}_{\tau}(\lambda, \gamma)\right)\right) \geq \gamma$

But $\rho=\rho \wedge(\mu \vee \lambda) \leq \rho \wedge\left(C_{\tau}(\mu \vee \lambda, \gamma)\right)$, and we have

$\mathrm{I}\left(\rho \backslash \operatorname{Int}_{\tau}(\mu \mathrm{V} \lambda, \gamma) \leq\left(\rho \wedge \mathrm{C}_{\tau}(\mu \mathrm{V} \lambda, \gamma)\right) \backslash \operatorname{Int}_{\tau}(\mu \mathrm{V} \lambda, \gamma) \leq\left(\rho \wedge \mathrm{C}_{\tau}(\mu \mathrm{V} \lambda, \gamma)\right) \backslash\right.$ $\left.\left(\operatorname{Int}_{\tau}(\mu, \gamma) \vee \operatorname{Int}_{\tau}(\lambda, \gamma)\right)\right) \geq \gamma$. is $r$-gflo.

Hence, $\rho \backslash \nu \leq \operatorname{Int}_{\tau}(\mu \vee \lambda, \gamma)$ for some $I(v) \geq \gamma$. This proves that $\mu \vee \lambda$

\section{Corollary 1.1}

Let $(\mathrm{X}, \tau, \mathrm{I})$ be an fuzzy ideal topological space, $\mu, \lambda, \in \mathrm{I}^{\mathrm{X}}$ and $\mathrm{r} \in \mathrm{I}{ }_{0}$. If $\mu$ and $\lambda$ are r-gflo sets, $\underline{1}-\mu$ and $\underline{1}-\lambda$ are fuzzy separated. Then $\mu \wedge \lambda$ is $r$-gflc.

Proof

Obvious.

\section{Corollary 1.2}

Let $(X, \tau, I)$ be an fuzzy ideal topological space, $\mu, \lambda, \mathbf{I}^{\mathrm{X}}$ and $\mathrm{r} \in \mathrm{I}_{0}$. If $\mu$ and $\lambda$ are r-gflo sets, then $\mu \wedge \lambda$ is $r$-gflo.

\section{Proof:}

Obvious.

\section{Theorem 2.5}

Let $(\mathrm{X}, \tau, \mathrm{I})$ be an fuzzy ideal topological space, $\mu, \lambda, \in \mathrm{I}^{\mathrm{X}}$ and $\mathrm{r} \in \mathrm{I}_{0}$. If and $\mu \leq \lambda$, and $\mu$ r-gflo relative to $\lambda$ and $\lambda$ is $r$-gflo relative to $X$, then $\mu$ $\mathrm{r}$-gflo relative to $\mathrm{X}$.

\section{Proof}

Suppose that $\mu \leq \lambda, \mu$ is $\mathrm{r}$-gflo relative to $\lambda$ and $\lambda$ is $\mathrm{r}$-gflo relative to X. Let $\rho \leq \mu$ and $\tau(\underline{1}-\rho) \geq \gamma$. Since $\mu$ is r-gflo relative to $\lambda$. By Theorem 2.5. $\rho \backslash v_{1} \leq \operatorname{Int}_{\lambda}(\mu, \gamma)$ for some $\mathrm{I}_{\lambda}\left(v_{1}\right) \geq \mathrm{r}$. This implies that there exists $\tau\left(\omega_{1}\right) \geq \gamma$ such that

$$
\rho \backslash \nu_{1} \leq \omega_{1} \wedge \lambda \leq \mu,
$$

for some $\mathbf{I}_{\lambda}\left(v_{1}\right) \geq \lambda$. Let $\rho \leq \lambda$ and $\tau(\underline{1}-\rho) \geq \gamma$. Since $\lambda$ is r-gflo, we have

$$
\rho \backslash v_{2} \leq \operatorname{Int}_{\tau}(\lambda, \gamma)
$$

for some $\mathrm{I}\left(v_{2}\right) \geq \gamma$. This implies that there exists $\tau\left(\omega_{2}\right) \geq \mathrm{r}$ such that

$$
\rho \backslash v_{2} \leq \omega_{2} \leq \lambda,
$$

for some $\mathrm{I}\left(v_{2}\right) \geq \gamma$. Now

$$
\rho \backslash\left(v_{1} \vee v_{2}\right)=\left(\rho \backslash v_{1}\right) \wedge\left(\rho \backslash v_{2}\right) \leq \omega_{1} \wedge \omega_{2} \leq \omega_{1} \wedge \lambda \leq \mu .
$$

This implies that $\rho \backslash\left(v_{1} \vee v_{2}\right) \leq \operatorname{Int} \lambda(\mu, \gamma)$ for some $\mathrm{I}\left(v_{1} \vee v_{2}\right) \geq \gamma$.

Thus, $\mu$ r-gflo relative to $\mathrm{X}$.

\section{References}

1. Chang CL (1968) Fuzzy topological spaces. J Math Anal Appl 24: 182-190.

2. Chattopadhyay KC, Hazra RN, Samanta SK (1992) Gradation of openness: fuzzy topology. Fuzzy sets and Systems 49: 237-242.

3. Chattopadhyay KC, Samanta SK (1993) Fuzzy topology: fuzzy closure operator, fuzzy compactness and fuzzy connectedness. Fuzzy sets and Systems 54: 207-212.

4. El Naschie MS (1996) Rossler oed G. Information and diffusion in quantum physics. Chaos, Solitons and Fractals 7(5).

5. EI Naschie MS (1998) On the uncertainty of Cantorian geometry and the twoslit experiment. Chaos, Solitons \& Fractals 9: 517-529.

6. El Naschie MS (2000) On the unification of heterotic strings, M theory and $E(\infty)$ theory. Chaos, Solitons \& Fractals 11: 2397-2408.

7. El Naschie MS (2004) A review of E infinity theory and the mass spectrum of high energy particle physics. Chaos, Solitons \& Fractals 19: 209-236.

8. El Naschie MS (2004) Quantum gravity from descriptive set theory. Chaos, Solitons \& Fractals 19: 1339-1344.

9. El Naschie MS (2004) Quantum gravity, Clifford algebras, fuzzy set theory and the fundamental constants of nature. Chaos, Solitons \& Fractals 20: 437-450.

10. El Naschie MS (2004) The symplictic vacuum, exotic quasi particles and gravitational instanton. Chaos, Solitons \& Fractals 22: 1-11.

11. El Naschie MS (2005) On a fuzzy Kähler-like manifold which is consistent with the two slit experiment. INT J Nonlin Sci Num 6: 95-98.

12. El Naschie MS (2006) Topics in the mathematical physics of E-in nity theory. Chaos, Solitons Fractals 30: 656-663.

13. El Naschie MS (2006) Elementary prerequisites for E-infinity (recommended background readings in nonlinear dynamics, geometry and topology). Chaos, Solitons \& Fractals 30: 579-605.

14. El Naschie MS (2006) Advanced prerequisite for E-infinity theory. Chaos Solitons \& Fractals 30: 636-641.

15. Kim YC, Ko JM (2004) R-generalized fuzzy closed sets. J Fuzzy Math 12: 7-22.

16. Saber YM, Abdel-Sattar MA (2014) Ideals on Fuzzy Topological Spaces. Appl Math Sci 8: 1667-1691.

17. Ramadan AA (1992) Smooth topological spaces. Fuzzy sets and Systems 48 371-375. 
Citation: Saber MY (2018) R-Generalized Fuzzy Closed Sets with Respect to an Ideal in Fuzzy Topological Spaces. J Appl Computat Math 7: 392. doi: 10.4172/2168-9679.1000392

Page 4 of 4

18. Ramadan AA, Abbas SE, Kim YC (2001) Fuzzy irresolute mappings in smooth fuzzy topological spaces. J Fuzzy Math 9: 865-878.

19. Šostak AP (1985) On a fuzzy topological structure. In Proceedings of the 13th Winter School on Abstract Analysis, pp: 89-103.
20. Zahran AM, Abbas SE, El-baki SA, Saber YM (2009) Decomposition of fuzzy continuity and fuzzy ideal continuity via fuzzy idealization. Chaos, Solitons \& Fractals 42: 3064-3077.

21. Zahran AM, El-Baki S, Saber YM (2009) Decomposition of fuzzy ideal continuity via fuzzy idealization. Int J Fuzzy Log Intell Syst 9: 83-93. 